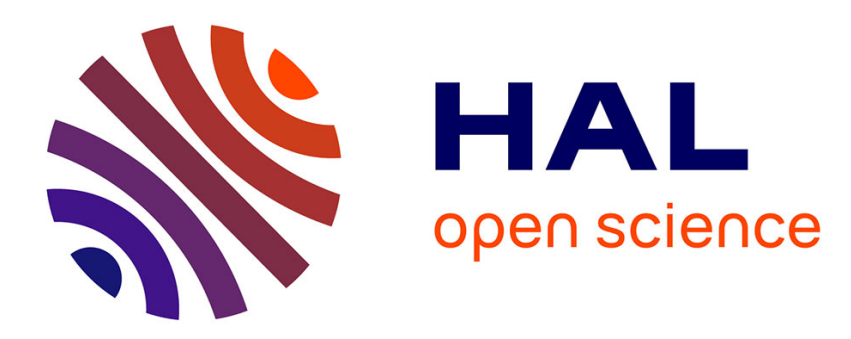

\title{
Infatigable Jean-Claude RAMEAU
}

Frédéric Mortier

\section{To cite this version:}

Frédéric Mortier. Infatigable Jean-Claude RAMEAU. Revue forestière française, 2006, 58 (2), pp.114118. 10.4267/2042/5809 . hal-03449457

\section{HAL Id: hal-03449457 https://hal.science/hal-03449457}

Submitted on 25 Nov 2021

HAL is a multi-disciplinary open access archive for the deposit and dissemination of scientific research documents, whether they are published or not. The documents may come from teaching and research institutions in France or abroad, or from public or private research centers.
L'archive ouverte pluridisciplinaire HAL, est destinée au dépôt et à la diffusion de documents scientifiques de niveau recherche, publiés ou non, émanant des établissements d'enseignement et de recherche français ou étrangers, des laboratoires publics ou privés. 


\title{
Infatigable Jean-Claude Rameau
}

\author{
FrÉdÉRIC MORTIER
}

\section{L'HOMME ET LA FORÊT}

Le Professeur Jean-Claude Rameau est connu de tous les forestiers français : un fait, je crois, unique pour un universitaire, enseignant-chercheur. Une référence. La Flore forestière française est la "flore de Rameau" ${ }^{(1)}$, l'atlas des habitats naturels forestiers est le classeur "Rameau", l'expert des catalogues de stations, le spécialiste de la dynamique forestière, de la protection de la biodiversité forestière... c'est Jean-Claude Rameau.

Surprenante sa présence sur le terrain, des milliers de forestiers ont fait des tournées et des formations avec lui et vous en parlent comme d'un moment exceptionnel, un privilège, une rencontre passionnante. La science de l'homme est immense comme sa vision globale et claire des écosystèmes forestiers et de leur dynamique. Tout aussi immense est sa simplicité et sa facilité de contact, ne râlant même pas quand il y a seulement du chou-fleur cru et une pomme pour déjeuner assis sur un rocher, pour peu qu'il y ait du café.

Lundi.

Jean-Claude Rameau est là sur la montagne de Lure en bras de chemise au mois de février malgré le froid. Il est bien sûr parti le dimanche matin en voiture de Nancy pour ne pas empiéter sur la semaine de travail. Le colosse à la voix incomparable captive le groupe de forestiers publics et privés en formation, qui le serrent de près, sur les stations forestières et la dynamique de la végétation. Comme un chien de sang, il a l'œil rivé au sol pour dénicher la pépite végétale à ne pas louper et qu'il tient absolument à commenter et à partager. II prend le temps de s'arrêter pour une lecture du paysage où la magie du maître opère vite sur ses auditeurs, les pièces du puzzle s'agencent impeccablement et tout devient logique et évident. Inlassablement ébahi de la beauté du paysage, il lâche, les mains dans les poches, " c'est pas mal!".

Mardi.

Il arpente la forêt domaniale du Boscodon avec des élèves GREF et mastères où il secoue amicalement les traînards en s'arrêtant net ébloui par une minuscule violette qu'il pince délicatement entre ses doigts de géant en mettant genou à terre comme pour mieux lui témoigner son respect. La leçon tonique du jour est la sapinière. Il apostrophe un élève tout affairé à autre chose: " $Y$ va écouter cet animal. Monsieur... aurait-il l'obligeance de nous faire part de ses commentaires?».

\section{Mercredi.}

Il est au bois du Chapitre en forêt de Gap-Chaudun où il encadre un élève de DEA. Ravi d'observer les stigmates de la sylvigénèse d'une forêt inexploitée depuis 50 ans, il repère vite également un ancien pré d'alpage, s'exclamant " y a du monde ici » quand il a identifié au moins dix espèces protégées sur moins de $20 \mathrm{~m}^{2}$.

(1) Nous ne faisons que constater cette dénomination populaire. Elle conduit à éclipser dans la bouche du public les autres auteurs de cet ouvrage collectif : voir plus de détails dans la contribution de Gérard Dumé (p. 104) (NDLR). 
Jeudi.

Il est au Siège de l'ONF avec le directeur général à qui la ministre de l'Environnement a demandé des solutions pour relancer la démarche Natura 2000 après le gel de la mise en œuvre décidé par le Premier ministre. De nombreux acteurs importants de tous horizons sont présents. Il défend avec conviction l'impérieuse nécessité d'investir dans la concertation, de donner des moyens aux CSRPN ${ }^{(2)}$, de prôner la gestion intégrée dans les sites Natura 2000. "Y nous emm... les écolos, y a pas de besoin de réserves partout, faut faire de la gestion intégrée avec des mosaïques, des lisières et des îlots de vieillissement "lâche-t-il devant l'assemblée en lissant sa cravate. L'intervention d'un collègue d'une ONG (3), pourtant sa culture d'origine, ne lui convient pas, il le montre ostensiblement à ses complices en tapotant sur la table, le menton levé, le regard en coin.

Vendredi.

Matin : Réunion à l'IDF ${ }^{(4)}$ au sujet de la flore forestière. "Je suis en retard", dit-il à voix basse comme un élève qui n’a pas repassé sa leçon, mais dans trois mois c'est terminé... "Salut à toi, bonjour à la famille ».

Après-midi : Réunion au ministère chargé de l'Environnement avec le Muséum sur les cahiers d'habitats forestiers. Soir : retour à Nancy.

Samedi.

ENGREF - Bureau dès 6 h 30 (au plus tard), "Faut bien déblayer les paperasseries de la semaine ». Rendez-vous successifs avec les thésards et les élèves dont il encadre les travaux. Des courriers à rédiger pour la Guyane, l'Iran, le Brésil, la Grèce, le CNPN (5), un conservatoire botanique et un commentaire d'article à faire sur les mégaphorbiaies et les forêts alluviales d'Alsace.

Dimanche.

Préparation d'un cours de phytoécologie forestière et d'une conférence sur la dynamique de la végétation pour l'ONF de Franche-Comté le matin et, après un bref déjeuner en famille, départ pour les forêts du Chili...

Pour bien comprendre l'agenda de ce passionné, bourreau de travail, il faut savoir que JeanClaude Rameau ne sait pas dire non quand il s'agit du service à rendre à la forêt, à un collègue, ou à un forestier.

Il a des vacances d'enseignant direz-vous ? Oui, mais particulières. Elles se passent dans des forêts à prospecter, savamment choisies, où son épouse, Françoise, et sa caravane (au barycentre du secteur) l'attendent patiemment. En visite dans la famille, Jean-Claude Rameau est un invité peu encombrant. Son beau-frère me confie un jour, qu'il était venu en famille à La Martinique, ils ne l'ont qu'aperçu durant deux semaines. Il partait tôt le matin jusqu'au soir à la découverte des forêts sèches, mésophiles et hygrophiles de l'île aux fleurs. Cela m'a d'ailleurs bien aidé quand j'étais aménagiste de la forêt des Pitons du Carbet et de la Montagne Pelée...

(2) CSRPN : Comité scientifique régional du Patrimoine naturel.

(3) ONG : Organisation non gouvernementale.

(4) IDF : Institut pour le Développement forestier.

(5) CNPN : Conseil national de la Protection de la Nature. 


\section{UNE GUVRE CONSIDÉRABLE QUI MARQUERA LONGTEMPS L'ÉCOLOGIE FORESTIĖRE EN FRANCE}

\section{Une œuvre écrite largement dédiée aux gestionnaires forestiers}

Pour Jean-Claude Rameau, l'avancée des connaissances ne vaut que si celles-ci sont diffusées, partagées et prises en compte par les gestionnaires. Il veille à ce que les documents soient pédagogiques, didactiques, aisément accessibles pour les non-spécialistes. Son œuvre porte principalement sur la typologie des stations forestières, la botanique, la phytoécologie forestière, la biodiversité forestière et les habitats naturels (Corine biotopes, Natura 2000...).

En chiffres, cette œuvre représente : 3 livres (Flore forestière française), 6 cartes de végétation, 2 volumes publiés par la Documentation française (habitats forestiers), 3 classeurs (biodiversité et gestion forestière) dont 2 pour la France, 1 pour la Belgique et le Grand-Duché du Luxembourg, 4 chapitres de livres publiés à plusieurs auteurs, 52 articles techniques ou scientifiques, 37 rapports d'expertises (stations, habitats naturels, biodiversité, impact des infrastructures, paysages) commandés par l'État ou des institutions publiques (parcs nationaux, parcs naturels régionaux, conservatoire du littoral, EDF, GDF...), 48 communications à des colloques, 10 congrès et excursions scientifiques de niveau national et international organisés ${ }^{(6)}$...

Le succès de ces ouvrages dans le monde forestier se résume à une recette évidente, mais dont il a le secret : marier la théorie et la pratique dans le souci de fournir des outils simples et d'accès facile au praticien.

\section{UN ENGAGEMENT TOTAL DANS LA FORMATION ET LA VULGARISATION}

Ancien instituteur, licencié en sciences naturelles, professeur certifié de collège puis de lycée en sciences naturelles, docteur de $3^{\mathrm{e}}$ cycle et docteur d'État, professeur de l'enseignement supérieur de classe exceptionnelle, Jean-Claude Rameau est rompu à la pédagogie et à l'enseignement de la science ; plus qu'un engagement c'est une vocation.

Deux phrases de Jean-Claude résument bien ses motivations.

Formation initiale : "L'école est la clé pour faire passer les messages aux futurs responsables ».

Formation continue : " Il faut vulgariser, il faut aider les gestionnaires et les propriétaires, cela ne sert à rien d'inscrire des espèces sur des listes protégées, si les acteurs ne se les approprient pas ».

Entre 1983 et 2005, c'est toute une génération d'ingénieurs forestiers, d'IGREF, de mastères, de thésards, d'étudiants en maîtrise ou DEA et d'élèves-techniciens qui a été sensibilisée et marquée par Jean-Claude Rameau à la prise en compte de la protection de la nature et de la dynamique de la végétation dans la gestion forestière. En approfondissement, le professeur Rameau a encadré plus de 180 étudiants stagiaires ou doctorants (ENGREF, autres écoles d'ingénieurs, universités françaises et étrangères, lycées agricoles et forestiers).

Les documents pédagogiques toujours clairs, précis, illustrés et commentés de Jean-Claude Rameau sont aussi nombreux que variés (botanique, autécologie, typologie des stations, gestion patrimoniale, écologie forestière, reconstitution post-tempêtes, dynamiques végétales...) : 33 références répertoriées (brochures, cédéroms) dont des manuels de 50 à 400 pages.

(6) Cf. liste des principales publications de J.-C. Rameau (p. 119). 
Le nombre de formations professionnelles qu'il a dispensées est incalculable : ONF, CRPF, IDF, CETEF, CFA, ATEN, ONCFS, DIREN, EDF, DRE (7), Sociétés d'autoroutes...

\section{UN ENGAGEMENT AUPRÈS DES INSTANCES OPÉRATIONNELLES ET DE DÉCIDEURS}

Son rang d'expert de haut niveau et son engagement pour la gestion des milieux naturels font qu'il est sollicité pour prendre des responsabilités dans des assemblées collégiales ou des fonctions de conseiller ou de président :

- conseil d'administration (parc national des Cévennes),

- conseils scientifiques (ONF, 8 conservatoires botaniques, 3 parcs nationaux, 2 parcs naturels régionaux, 1 réserve naturelle, 1 conservatoire de sites),

- groupes nationaux (typologie forestière, réseau écologique forestier, conservation des ressources génétiques forestières, loup, habitats naturels),

- ministère chargé des Forêts (circulaire "biodiversité et gestion forestière"),

- direction générale de l'ONF (instructions "biodiversité", "réserves", “Natura 2000", examen des propositions de sites Natura 2000 en forêt publique),

- groupe d'experts "habitats naturels" (Muséum national d'Histoire naturelle et ministère chargé de l'Environnement).

Jean-Claude Rameau est aussi président de la zone biogéographique continentale pour l'application de la directive européenne "habitats, faune, flore" et chargé auprès des CSRPN d'une animation pour appuyer l'élaboration de réponses régionales à la mise en œuvre de la directive.

\section{UNE PENSÉE ET UNE ACTION NOVATRICES ET DYNAMISANTES}

\section{Acte 1 : De l'affrontement à la concertation}

Les relations de Jean-Claude Rameau avec les forestiers sont d'abord tendues voire parfois conflictuelles dans les années 1970 en Haute-Marne. Mais chacun écoute et tient compte des contraintes et demandes de l'autre. Jean-Claude Rameau excelle dans l'exercice de concertation, qui sera un fil conducteur de son action, il cherche à comprendre les ressorts des acteurs tout en cherchant à les convaincre en leur apportant de l'information sur sa spécialité et leur montrer qu'il suffit d'un peu de précaution, en général, pour conserver le milieu ou l'espèce précieuse. Et la synergie opère. "Rameau", tel qu'il est appelé chez les forestiers, devient l'expert, puis la référence et l'allié qui est consulté sur le micro-projet local ou la définition d'une politique nationale. Il est un de ces scientifiques, qui osent "sortir du cadre" et "se mouiller" dans des dossiers opérationnels de terrain. Pour autant, il dit clairement et sans détour ce qu'il pense à ses interlocuteurs, de l'ouvrier forestier au ministre. Il a montré la voie...

\section{Acte 2 : De la protection sous cloche à la gestion intégrée}

Alors que le débat est nourri entre les enjeux de protection de la nature et de production de bois, et connaissant bien la gestion forestière, il prône, dès 1987, la gestion intégrée qui concilie les deux fonctions. Sa réflexion repose sur une logique implacable. L'essentiel de la biodiversité se situe en dehors des espaces protégés, les écosystèmes sont transformés par l'homme depuis

(7) ONF : Office national des Forêts ; CRPF : Centre régional de la Propriété forestière; CETEF : Centre d’Études techniques forestières ; CFA: Centres de Formation pour Adultes; ATEN: Atelier technique des Espaces naturels; ONCFS : Office national de la Chasse et de la Faune sauvage; DIREN : Direction régionale de l'Environnement ; DRE : Direction régionale de l'Équipement. 
le Néolithique, c'est donc par une diversité de modes de gestion et des corridors écologiques que la biodiversité dans toutes ses composantes pourra être conservée. Dans les cas où la fonction de protection est incompatible avec d'autres (sites à haute valeur patrimoniale), il propose de créer des réserves et de les gérer activement (suivi scientifique et génie écologique). Sa réflexion trouvera un prolongement concret dans Natura 2000 dont l'objectif est de concilier la protection de la nature et les activités des hommes. Jean-Claude Rameau sera un des contributeurs scientifiques majeurs de la mise en œuvre de la directive "habitats".

\section{Acte 3 : De l'approche statique à l'approche dynamique}

«Les phytosociologues doivent progresser, il faut intégrer les dynamiques ». Jean-Claude Rameau n’hésite pas à bousculer les dogmes (le fameux concept du climax) et les idées reçues (homéostasie, séries de végétation...). Il est parmi les pionniers en France qui lancent, dès 1985, les études sur les dynamiques linéaires en forêt (déprise agricole, déprise forestière) et les dynamiques cycliques de forêts en évolution libre (sylvigénèse). Son objectif : bien connaître les processus dynamiques pour les principales essences dans les grands groupes stationnels afin d'enrichir la gestion forestière et façonner des forêts plus diversifiées, plus stables et plus résilientes. Il a entamé la rédaction de 4 volumes à ce sujet en réponse à une commande du directeur technique de l'ONF.

\section{Acte 4 : Du règlement au contrat, du secret à la transparence}

Jean-Claude Rameau estime insuffisantes et d'efficacité limitée les mesures réglementaires de protection de la nature. "Les acteurs de terrain doivent les comprendre, se les approprier, ils doivent être associés à la définition des mesures de gestion et bénéficier de compensations en cas de préjudice ». Il s'engouffre avec entrain dans l'ère des contrats " cela donne du corps et une réalité concrète à la protection de la nature ». Il milite aussi pour la mise à disposition des données naturalistes auprès des gestionnaires, aménageurs et décideurs. "La plupart des erreurs sont faites par défaut d'information et de connaissance, non par mauvaise volonté » s'exclame-t-il.

\section{ÉPILOGUE ET PRÉAMBULE}

L'héritage et les services rendus par Jean-Claude Rameau sont à la mesure du personnage : énormes et profondément humains. Il a ouvert des voies avec le courage et l'enthousiasme des premiers de cordée. Il ne donnait pas de poissons, mais apprenait à pêcher. Il a dépensé son énergie sans compter, avec une force à déplacer les montagnes qu'il affectionnait tant. Il a beaucoup donné en se faisant plaisir et en travaillant dans la joie. Il nous revient collectivement de reprendre le flambeau et de suivre la voie tracée.

Merci Monsieur le Professeur Jean-Claude Rameau. Merci cher ami...

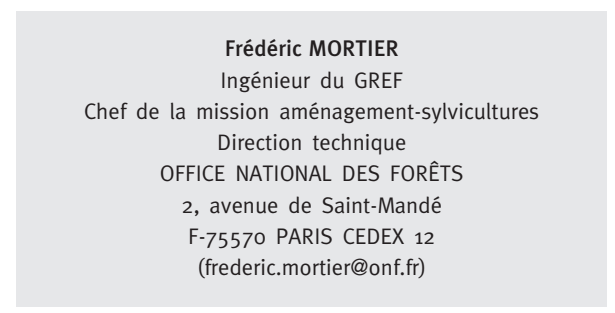

